\title{
ИСКУССТВОВЕДЕНИЕ
}

UDC 72

DOI: $10.24044 / \mathrm{sph} .2017 .1 .15$

\section{IMPLEMENTATION OF INTERACTIVE ENVIRONMENT FOR RECOVERY AND REHABILITATION}

K. Boychenko
Master

Polytechnic University of Milan

Milan, Italy

\begin{abstract}
The article describes impact of interactive environment or its' elements on recovery in medical field. It explains benefits of interactive systems embedded on design stage and how these systems can improve users' or patients conditions in future, helping them to recover faster, changing their experience of medical environment and recovery process. The research considers relation between space perception and attachment to the space, explaining how interactive environment effect patients on mental, emotional, physical and even social scales. The article proves that interactive environment, virtual reality and smart objects are able to provide space with new qualitative characteristics.
\end{abstract}

Keywords: interactive environment; architecture; smart design; recovery; space perception; virtual reality.

\section{Introduction}

It's a well known fact that the environment plays a major role in recovery of patients after surgery, while on treatment or those who have to face with rehabilitation in their everyday life. For this kind of people interactive and responsive environment is not just a relief in comparison with ordinary 4 white walls of the hospital, but also a tool to recover faster and make the process more pleasant. The patients are not necessarily obliged to see all the frightening tools and sensors all over them, feeling anxious and uncomfortable under the cold hospital lamps' light. With application of interactive techniques, the patients may not even be aware of all the data gathered from a session and the hidden procedures running during their presence in the room. They will receive maximum relaxation and minimum reminding of the reason they are in a medical facility.

During the design of the building, evaluation and prediction of users' need has to be taken into consideration, as it often might require a specific organization of the system, and the needs are changing over time. The primary targets for development of the space' ac- 
tivities are the military, the elderly and the handicapped.

Benefits of interactive architectural solution include the possibility for physical therapy, exercise, and other activities that are beyond the typical demands of the living space. Interactive technologies can allow disabled and older people to retain desired level of independence within their home that previously was impossible. People with reduced capabilities may experience difficulties with performing everyday activities, like opening doors, windows, moving inside their living space and so on. It is now possible to make their life much easier and comfortable by means of interactive architecture.

2. Literature data analysis and problem statement

Motion in architecture already has been discussed many times by various professionals (Giedion, Ferstegen, Jormakka, Lynn) in attempts to describe, classify, separate or represent it. Motion is one of the driving forces aiming to express architecture. There are two main methods of expressing motion in architecture: the procession or the superimposition. In processional models of time, architecture is the immobile frame through which motion passes. It is based on static frames and have fixed relationships between functional program and user. The elimination of force and motion from form is the basis of recent alternatives (e.g. sequential model). Static frames are being multiplied and put in a sequence in order to introduce the idea of "dynamic" architecture as multiply framed. An alternate model of time and motion resists the separation of form from its animating forces. Form is perceived in a space of virtual movement and force rather than within an ideal equilibrium space. Instead of fixed prototype, a flexible, mutable models are created, which are rather a potential of multiple variables ("performance envelope"). In result "architecture can be modeled not as a frame but as a mobile participant in dynamical flows" (Lynn, 1998). To do that, necessary is more than a shift in technology, but rather a shift in sensibility from reduction to combination to compose time based, topological designs. Although the introduction of time and motion techniques into architecture affects visual qualities, it is inappropriate to understand technology in terms of style.

With the development of new technical capabilities, "smart objects" start being able to receive a lot of information from the external environment. The people's behavior is a significant part of this environment. This leads to necessity of understanding the feedback with the world and the notion of "family of smart objects" in our environment that can communicate with us and with each other by sending messages using the built-in microprocessors which are giving different information. A lot of these components are designed to capture information from the environment, such as temperature, light levels, wind speed and noise. Some components are able to receive simple messages from the man. They feel our presence radiated heat or movement and react in some way [1] 
Any action transfers information. Oosterhuis argues that modern architects design structures to shape the behavior of the media. It is so for the very simple house. Urban planners design strategies for structuring the information flow in the city. It is so for all cities, large or small. Oosterhuis offers to pay more attention to the membranes in the spaces and passages in these membranes within the design process, taking into account the flow of information in any form, instead of focusing on the physical exterior spaces that are built upon ideas about the behavior of people.

\section{Purpose and objectives of re- search}

The social conditions change with the progress of new technologies and so do the building typologies, becoming interchangeable and developing a universal type of space that is able to accommodate various typologies at the same type. This new type of space requires specific mobility and flexibility.

Interactive Architecture may be introduced as convergence of artificial intelligence on one side and kinetics on the other. The intelligence is represented by embodied computation and balanced by the physical part (kinetic) in order to create the architectural environment that would successfully satisfy the evolving needs and psychological, physical, social and environmental demands. It uses modern and constantly developing technologies, exploring new methods and applications that enable the space to reach new qualities, becoming dynamic, respond- ing and active. The kinetic function is performed by the ability of moving object to create adaptable spatial variations or the motion of transformable objects, occupying the predefined space. Robert Kronnenberg defines such systems as buildings or building components with variable mobility, location, and/or geometry [10].

Intelligent space may be considered as a reactive and interactive space that is able to analyze and understand its environment or conditions, operate received information, learn from it and produce a feedback.

The types of pressure on trends in kinetic architecture can be divided into two general categories: "pragmatic" and "humanistic". The implication of kinetic architecture touch upon building performance on the one hand and aesthetic phenomenology on the other. Pragmatic applications are concerned with solving needs and optimizing solutions. The general implications of utilizing such systems in architecture include, but are not limited to: space efficiency, shelter, security, transportation, safety, and economics. The humanistic pressures define physical and psychological the effects that the changes in architectural environment has on its users. It means that the motion of architectural elements should be designed considering the way a user, that might move as well, would perceive this space, with constantly changing vantage point. Chuck Hoberman points the emotional dependence on the motion: "When one sees this special behavior [of transfor- 
mation], one feels it in one's body perhaps a physiological connection, because there is a sensation, a physical sensation and a mental perceptual sensation." For instance, temporary divisions in hospitals, movable partitions in offices or furniture that disappears or transforms when it is not in use are driven by pragmatic need for privacy rather that by humanistic needs [11].

\section{Research results}

People with limited abilities (due to current state for a short period of time till they are able to perform everyday tasks by themselves or due to their general health conditions) need special assistance and it is not always desired to get it from other people or to ask for it. They also need therapy that may scare and depress, experienced as a torture. In many cases of post-surgery therapy patient need to do special exercises throughout a long period of time, that are often confusing, not saying hard to perform. The patients can start losing their motivation as a result of being not able to do the exercises correctly from physiological point of view and to achieve a necessary relaxation psychologically. Consequently, physiological and psychological problems increase the risk of backset of the disease or slow down the recovery progress significantly.

A major part of the patients need more support for their rehabilitations to avoid possible complications, including physically disorder, attention and memory diminished due to stress, anxiety, fatigue and nausea, mitigate side effects and recover their wellbeings. The technological develop- ment of responsive space is able to improve patients' physical therapy and psychological relaxation experience through careful applications of interactive technologies. It allows to make the whole procedure fun and playful for patients, like a game they are willing to play it and to exercise every day, to distract them from pain, discomfort and depressing thoughts, to make the procedures personalized for each visitor and adjusted to his or her special needs, and to help the medical personnel in making more accurate observations of patients' recovery progress and maintain safety.

If user can adjust physical geometry, the whole new field of possibilities opens in the way the environment can be used in real time. This new experience relies on understanding and interpretation of potential of the space, it's components, in a specific time frame. Architecture now plays a more active role in suggesting new ways for its' users, based on real time information exchange and adaptation. This experience depends on the way a user or a group of users decide to interact with it, whereby the rules are dynamic and variable [4].

Interactive architecture, in terms of relying on sympathetic understanding of biological systems, may be considered as a breakthrough in the old methods of its' organization and construction. Space with high communicative capability can foster a heightened sense of attachment. According to Salingaros: "Our society tries to understand its own structure, and builds its physical extensions on the earth's sur- 
face, guided by the blank slate hypothesis" [8].

The real attachment to space is only possible when the space becomes truly interactive, understanding itself and the users, able to sustain, replicate or even enhance the features that make it special on the personal levels. When the space understands and mediates all the multiple (sometimes conflicting) desires and needs, the users start to understand the space by means of anthropomorphic concepts, like supporting or understanding. This space creates strong attachment to itself.

\section{Research methodologies and materials}

In previous years multimedia technology is usually applied to help patients' in gaining all the information they need about their diseases and ways to treat and fight them. Now the technology is used to persuade patient to do exercises, and to stimulate their ability in paying attention on learning rehabilitations. For example, a computer aided virtual reality simulations for stroke patients to rediscover their motor capabilities. The studies show that the task of learning in a multimedia system can really help stroke patients recover their physical capabilities, shortens the therapeutic time, and encourages patients to do the exercises in a more positive way [7].

The Rehabilitation Gaming System (RGS) is one of many examples of interactive environment implication for rehabilitation purposes. It has been designed as a flexible, virtual-reality based device for rehabilitation of neu- rological patients. The RGS is based on the neurobiological considerations that brain plasticity remains throughout life and can thus be utilized to achieve functional reorganization of areas affected by stroke by means of activation of secondary motor areas such as the so called mirror neurons system. As a multi-level adaptive tool, the RGS provides a task oriented game training with individualized graded complexity. It is assumed that the VRbased training protocol related to RGS creates conditions that aid recovery by virtue of the human mirror neuron system. The evidence for this assumption is identifying the brain areas involved in controlling the catching of approaching colored balls in the virtual environment of the RGS. The imagery of target catching was related to activation of frontal, parietal, temporal, cingulate and cerebella regions. These activations in relation to object processing, attention, mirror mechanisms, and motor intention. Active catching followed an anticipatory mode, and resulted in significantly less activity in the motor control areas [9].

\section{Research discussion}

The environment should help the patients, especially in the beginning of the rehabilitation process, when they fill nervous and unfamiliar with the procedure and space. It is able to create a friendly environment by analyzing the patients' heart-beat and breath rhythms and to help them in relaxation, shaping the rhythm with adjusted to each patient sound, light and temperature performance of the space. The 
embedded computation system will analyze the reactions of the patient and changes in his or her conditions, in order to create the most suitable environment. In breath relaxation sessions a respiratory sensor can monitor, for example, the movement of the abdomen region of the patient, this way the system will have the needed data about the status of the relaxation by visualizing the biofeedback results, and to recognize the patients' current relaxation level in real-time during the procedure. The preferred settings can be saved for later when this patient comes back.

For some types of therapy full body and mind relaxation is crucial, in this case a virtual reality room is a perfect tool. The virtual reality rooms can help patients in achieving maximum level of relaxation. While relaxing narrations persuade the patient to imagine a virtual journey, the screens combined with sound and light effects show changing video of the relaxing places, a green loan, a warm sea or the sky, the couch or a special chair emulates the movement, helping a patient to dive deeply into this illusory reality. For example the journey can take place in the outer-space full of stars, which will reflect the patients' breathing rhythm. With the data derived from respiration sensors embedded in the equipment secretly, the stars will move according to the speed and depth of the patients' exhalation and inhalation. This interactive performance can help the patients to leave their fears and problems in the endless cosmic space. In the end of the session the patient will be brought back through a beautiful nebula, and then taken back to earth eventually and safely grounding back to reality.

\section{Conclusion}

In interactive spaces designed by architects, technological development rise a discourse about the role of the architect, because now the spaceresponsive mechanisms allow people to choose a primary position and the configuration (the design) of their own space, taking into account the needs, allowing to change the color, texture, size, and transparency of the walls, offering a dialogue with the environment, making it clear that architects no longer obliged to constraint and limit boundaries of human movements and desires. A person becomes a co-author of a participant of development of architectural environment: on one hand, he transforms the state of the form, on the other hand - the response, the user now is largely responsible for the outward manifestation of the form. Such an environment interacts and responds to anyone who wishes to interact (the concept of "open source") from a child to a professor. There may be questions: what happens if the media is angry, what degree of freedom and independence it can have and what opportunities and limits of these possibilities belong to the user? [3]

Bertsky argues that our culture is supersaturated with effects, in other words, the entire production process is hidden from us. We are surrounded by effects. All magically appears from nowhere, only the result is exposed. Therefore, in this conditions the architecture should operate on the level of effects as well [12]. 
Ben van Berkel says that the role of architecture is to make us understand ourselves and the world around us in a new way, remain a mirror for the real world" [2;6].

Using emerging images, light, sound, moving and changing its spatial configuration intelligent space makes the user sink into the atmosphere that is created for each of the activities taken within it and able to project information in the outer shell. Smart environment proposes the whole situation for the user to get involved into this conversation with the space, full of information, hidden or exposed possibilities, choice of activities, details, games and even jokes and allusions that the user is free to intemperate as his intelligence, experience and sense of humor lets him to [5].

\section{Bibliography}

1. Alyena Kereshun, Abilities of Interactive Architecture, Architecton: university news № 14, 2006.

2. Ben van Berkel, Caroline Bos, Move, Paperback 1999.

3. Dana Cuff, Archtecture: the story of practice MIT Press 1991.

4. Dan Saffer, The Elements of Interaction Design, UXmatters ,05 2006.

5. Kristina Boychenko, Interactive Architecture: development and implementation into the built environment, European
Journal of Technology and Design Vol. (15) March 2017.

6. Kristina Boychenko, Sences and Behavior of Interactive Environment, Vestnik Nauki i Obrazovaniya №2 (26) February 2017.

7. National Taiwan Uniwersity, i-m-Space: Interactive Multimedia-Enhanced Space for Rehabilitation of Breast Cancer Patients" (Graduate Institute of Networking and Multimedia; Department of Computer Science and Information Engineering; College of Medical; Department of Psychology; National Taiwan University; Foundation of Breast Cancer Prevention and Treatment 2010.

8. Nikos A. Salingaros, Towards a Biological Understanding of Architecture and Urbanism: Lessons from Stephen Pinker, Katarxis 32004.

9. Prochnow D, Bermúdez I Badia S, Schmidt J, Duff A, Brunheim S, Kleiser R, Seitz RJ, Verschure PF, A functional magnetic resonance imaging study of visuomotor processing in a virtual realitybased paradigm: Rehabilitation Gaming System, Department of Neurology, University Hospital Düsseldorf, Düsseldorf, Germany 2009.

10. Robert Kronnenber, Transportable Environments 2, London, Spon Press 2003.

11. Transformable Architecture, Interview with Chuck Hoberman, PingMag, 07 2007.

12. Vladiir Belogolovskiy, Interview with Aaron Betsky, Architectural herald, №2 (89) 2006.

(C) Boychenko K., 2017. 